\title{
„MITTEILUNGEN DER VEREINIGUNG ÖSTERREICHISCHER BIBLIOTHEKARINNEN UND BIBLIOTHEKARE“ IN SHERPA/ ROMEO („THIS IS A ROMEO GREEN JOURNAL“) UND ERIH PLUS
}

\section{Liebe Leserinnen und Leser,}

wir freuen uns, Sie darüber informieren zu können, dass die Mitteilungen der Vereinigung Österreichischer Bibliothekarinnen und Bibliothekare als Open Access-Zeitschrift nunmehr auch als "green journal" in SHERPA/RoMEO aufgenommen wurden: http://www.sherpa.ac.uk/romeo/search. php?issn=1022-2588.

SHERPA/RoMEO ist ein Online-Verzeichnis, das Open Access-Policies von Verlagen weltweit sammelt und analysiert und auf der Ebene einzelner Zeitschriften - derzeit 3.074 Titel - Informationen bereitstellt, wie seitens der Verlage mit dem Urheberrecht der AutorInnen umgegangen wird und welche Möglichkeiten zur Selbstarchivierung angeboten werden. Dabei wird, je nachdem, ob die Selbstarchivierung von Preprints und Postprints (green), nur von Preprints (yellow), nur von Postprints (blue) oder gar nicht (white) erlaubt ist, in vier Kategorien unterschieden.

Überdies erfolgte im Februar 2017 auch die Aufnahme in den European Reference Index for the Humanities and the Social Sciences (ERIH PLUS): https:// dbh.nsd.uib.no/publiseringskanaler/erihplus/periodical/info?id=490377.

ERIH PLUS ist eine nicht-kommerzielle Initiative, deren Ziel es ist, durch die Bereitstellung einer internationalen Infrastruktur und einem umfassenden Angebot an bibliographischen Daten - zur Verbesserung der Sichtbarkeit von Veröffentlichungen aus den Geistes- und Sozialwissenschaften ( $\mathrm{SSH}$ ) beizutragen; ursprünglich von von Wissenschaftlerinnen und Wissenschaftlern unter dem Dach der European Science Foundation (ESF) initiiert, wird das Verzeichnis seit 2014 vom Norwegian Centre for Research Data betrieben.

Darüber hinaus bringt die erste Ausgabe der Mitteilungen der VÖB des Jahres 2017 wieder eine Fülle an Informationen zu verschiedenen Themen des Bibliothekswesens - von Neuigkeiten aus der VÖB über die Berufseinsteigersituation von Informationsexpertinnen und -experten bis zu Forschungsdatenmanagement und der Restitution von NS-Raubgut. 


\section{Aus der VÖB}

In der Kolumne „Aus Präsidium und Vorstand“ informiert Werner Schlacher über die jüngste Entwicklung der Vereinigung Österreichischer Bibliothekarinnen und Bibliothekare, insbesondere über den bevorstehenden 33. Österreichischen Bibliothekartag in Linz [S. 9].

Peter Graf und Alexander Zartl haben einen „Nachruf auf Wolfgang Kerber (1942-2017)“ verfasst, der von 1977 bis 2008 Direktor der Österreichischen Zentralbibliothek für Physik gewesen ist und in dieser Funktion bemerkenswerte Akzente für das österreichische Bibliothekswesen gesetzt hat. Wolfgang Kerber gelang es auch, Politikerinnen und Politiker für das Bibliothekswesen zu interessieren - so besuchten der damalige Bundeskanzler Bruno Kreisky 1989 und die damalige Außenministerin Benita Ferrero-Waldner 1997 die Zentralbibliothek - und auch den Pionier der Bibliometrie, Eugene Garfield, konnte er 2004 in der von ihm geleiteten Bibliothek begrüßen [S. 13].

„In Memoriam Harro Heim (1919-2016)“ schreibt Karl F. Stock über seine Erinnerungen an den langjährigen Bibliotheksdirektor der Universitätsbibliothek Bielefeld (1968 bis 1984). Harro Heim war über seine gesamte Berufslaufbahn dem österreichischen Bibliothekswesen sehr verbunden so hat er die österreichischen Bibliothekartage von den 1960er bis in die 1990 er Jahre regelmäßig besucht [S. 17].

Unter dem Titel „Die VÖB-Kommission Universitätsbibliotheken an österreichischen Privatuniversitäten stellt sich vor" informieren Ingo Glückler und Katharina Mrkvicka über die jüngste Kommission der VÖB [S. 21].

Abgeschlossen werden die Informationen zur VÖB mit den Einladungen zur 63. Generalversammlung, zur 1. Präsidiumssitzung bzw. zur 1. Vorstandssitzung für die Funktionsperiode 2017-2019, zur Wahl des Präsidiums für die Funktionsperiode 2017-2019 sowie zu Ehrungen [S. 24].

\section{Beiträge und Reports}

Die „K. (u.) k. Militärbibliotheken“ werden in einem Beitrag von Erik Gornik thematisiert. Auf Basis einer Analyse einer Bibliotheksstatistik der k. k. statistischen Zentralkommission von 1870 werden die verschiedenen militärischen Bibliotheken vorgestellt: Amtsbibliotheken, Bibliotheken der militärischen Bildungsanstalten, Bibliotheken militärischer Versorgungs-, Heil- und Badeanstalten, Bibliotheken der Truppenkörper sowie Militärvereinsbibliotheken [S. 27].

Unter dem Titel „Wohin mit den InformationsexpertInnen? Ergebnisse einer Erhebung zur Berufseinstiegssituation der AbsolventInnen des Grundlehrgangs Library 
and Information Studies" berichten Melanie Nowak und Clara Schneckenleithner über eine im Rahmen des ULG an der Österreichischen Nationalbibliothek entstandene Studie, die unter Absolventinnen und Absolventen des ULG der Jahre 2010 bis 2015 zu den Themen Arbeitsmarktperspektiven, Beschäftigungsverhältnisse und Berufszufriedenheit durchgeführt worden ist. Neben einer Online-Umfrage stützte sich die Studie auch auf eine Auswertung von Stellenbeschreibungen [S. 42].

Der Report „e-Infrastructures Austria 2016: Bericht über das dritte Jahr des Hochschulraumstrukturmittelprojekts für den koordinierten Aufbau und die kooperative Weiterentwicklung von Repositorieninfrastrukturen" wurde von Bruno Bauer, Paolo Budroni, Andreas Ferus, Raman Ganguly, Eva Ramminger und Barbara Sánchez Solís zusammengestellt. In den drei Teilprojekten dieses innovativen Projektes konnten wichtige Schritte gesetzt bzw. abgeschlossen werden, die vom Aufbau von Repositorien an den 25 Partnereinrichtungen über die Entwicklung von Muster-Policies für Forschungsdatenmanagement bis zu einem viertägigen Fortbildungsseminar für Forschungsdaten und e-Infrastrukturen reichten [S. 66].

\section{Mitteilungen}

Zum Thema „Universitätsbibliothek Salzburg restituierte Bücher und handschriftliche Manuskripte aus dem Konradinum in Eugendorf" wurde ein Beitrag von Irmgard Lahner verfasst. Die Restitution erfolgte in einem feierlichen Festakt am 31. Jänner 2017 [S. 94].

In den „Mitteilungen aus der OBVSG“ informiert Peter Klien über die Recherchemöglichkeit nach österreichischen Hochschulschriften in der OBV-Verbundsuchmaschine, über die Aufnahme der Bibliotheken der Diözese Linz in den Österreichischen Bibliothekenverbund, über den Abschluss des Umstiegs auf Primo Sharing sowie über die Ernennung von Bettina Kann zur neuen Leiterin der Abteilung „Verbundbetreuung und -koordination“ [S. 100].

Bruno Bauer, Markus Feigl, Gabriele Fröschl, Martina Griesser, Rainer Hubert, Lorenz Mikoletzky, Alfred Schmidt, Siegfried Steinlechner und Harald Wendelin berichten über „BAM Austria: Arbeitskreis Bibliotheken, Archive, Museen Österreichs: Aktivitäten 2016“ [S. 103].

David Frank informiert in seiner „Einladung zur Jahrestagung der Arbeitsgemeinschaft für Medizinisches Bibliothekswesen (AGMB) an die Veterinärmedizinische Universität Wien von 25. bis 27. September 2017 “ über die jährlich durchgeführte Informations- und Fortbildungsveranstaltung der mitgliederstärksten fachspezifischen Bibliothekarsvereinigung im deutschsprachigen Raum, die in diesem Jahr nach längerer Zeit wieder einmal in Österreich stattfinden wird [S. 107]. 


\section{Veranstaltungsberichte}

Michaela Zemanek berichtet über die „1. Informationskompetenz-Tagung Deutschland/Österreich", die am 16. und 17. Februar 2017 in Innsbruck stattgefunden hat [S. 111].

Im Rahmen dieser Tagung hat Jens-Peter Gaul einen Vortrag zum Thema „Informationskompentenz und Hochschulen - ein produktives Spannungsverhältnis" gehalten, den er freundlicherweise zur Veröffentlichung in den Mitteilungen der VÖB zur Verfügung bereitgestellt hat [S. 115].

Über die Tagung „Digitale Bibliothek. Zukunft des digitalen Erbes? Nachhaltige Lösungen für Gedächtnis- und Wissenschaftseinrichtungen" die am 2. und 3. März 2017 in Graz veranstaltet wurde, informieren Susanne Blumesberger und Veronika Gründhammer [S. 128].

\section{Rezensionen}

Die aktuelle Ausgabe der Mitteilungen der VÖB wird wieder vom Rezensionsteil abgeschlossen. Verfasst wurden die Rezensionen von Anna Krenn (Sitzen ist das neue Rauchen. Das Trainingsprogramm, um Haltungsschäden vorzubeugen und unsere natürliche Mobilität zurückzugewinnen [S. 134]), Werner Schlacher (Letzte Bibliotheken: Reflexe eines schwindenden Zeitalters [S. 136]), Renate Schönmayr (NS-Provenienzforschung und Restitution an Bibliotheken Praxiswissen [S. 137]), Rene Thalmair (Knowledge services. A strategic framework for the 21st century organization [S. 139]) und Sandra Zoglauer (Erfolgreich recherchieren - Altertumswissenschaften und Archäologie [S. 143]).

Wir wünschen Ihnen auch mit der vorliegenden Ausgabe 1/2017 der Mitteilungen der Vereinigung Österreichischer Bibliothekarinnen und Bibliothekare viel Spaß bei der Lektüre und viele nützliche Hinweise für Ihren beruflichen Alltag.

Ihr Redaktionsteam der Mitteilungen der VÖB

Beiträge und Feedback erbeten an: voeb-mitt@uibk.ac.at. 